\title{
A cryogenic beam of refractory, chemically reactive molecules with expansion cooling
}

\author{
Nicholas R. Hutzler, ${ }^{* a}$ Maxwell F. Parsons, ${ }^{a}$ Yulia V. Gurevich, ${ }^{a}$ Paul W. Hess, ${ }^{a}$ \\ Elizabeth Petrik, ${ }^{a}$ Ben Spaun, ${ }^{a}$ Amar C. Vutha,${ }^{b}$ David DeMille, ${ }^{b}$ \\ Gerald Gabrielse $^{a}$ and John M. Doyle ${ }^{a}$
}

Received 24th March 2011, Accepted 27th May 2011

DOI: $10.1039 / \mathrm{c1cp20901a}$

Cryogenically cooled buffer gas beam sources of the molecule thorium monoxide (ThO) are optimized and characterized. Both helium and neon buffer gas sources are shown to produce ThO beams with high flux, low divergence, low forward velocity, and cold internal temperature for a variety of stagnation densities and nozzle diameters. The beam operates with a buffer gas stagnation density of $\sim 10^{15}-10^{16} \mathrm{~cm}^{-3}$ (Reynolds number $\sim 1-100$ ), resulting in expansion cooling of the internal temperature of the ThO to as low as $2 \mathrm{~K}$. For the neon (helium) based source, this represents cooling by a factor of about 10 (2) from the initial nozzle temperature of about $20 \mathrm{~K}(4 \mathrm{~K})$. These sources deliver $\sim 10^{11} \mathrm{ThO}$ molecules in a single quantum state within a 1-3 ms long pulse at $10 \mathrm{~Hz}$ repetition rate. Under conditions optimized for a future precision spectroscopy application [A. C. Vutha et al., J. Phys. B: At., Mol. Opt. Phys., 2010, 43, 074007], the neon-based beam has the following characteristics: forward velocity of $170 \mathrm{~m} \mathrm{~s}^{-1}$, internal temperature of $3.4 \mathrm{~K}$, and brightness of $3 \times 10^{11}$ ground state molecules per steradian per pulse. Compared to typical supersonic sources, the relatively low stagnation density of this source and the fact that the cooling mechanism relies only on collisions with an inert buffer gas make it widely applicable to many atomic and molecular species, including those which are chemically reactive, such as ThO.

\section{Introduction}

Atomic and molecular beams are important tools for precision spectroscopy and collision studies. ${ }^{1,2}$ The most commonly used beam methods are effusive and supersonic (typically pulsed in the latter case). Effusive beams of certain species have very large fluxes, especially metal atoms or low-reactivity molecules with high vapor pressure. Often, however, these beams have large velocity spreads and broad distributions over internal states, reducing the useful signal for many spectroscopy experiments. Supersonic beams, on the other hand, are cold translationally and internally, but have a large forward velocity. Typically, for spectroscopy, it is desirable to have a beam that is both cold (to narrow spectral features, and, in the case of molecules, concentrate population in a small number of rotational levels) and slow (to increase the interaction time and decrease time-of-flight broadening) while maintaining high flux. There is a range of techniques to achieve such a beam. For example, beams of many atomic species can

${ }^{a}$ Harvard University Physics Department, 17 Oxford Street, Cambridge, MA 02138,USA.E-mail: hutzler@physics.harvard.edu;

Tel: + 16174952713

${ }^{b}$ Yale University Physics Department, 217 Prospect Street,

New Haven, CT 06511-8499, USA be laser-cooled and slowed using dissipative optical forces, ${ }^{3}$ through techniques such as frequency chirping, ${ }^{4}$ Zeeman slowing, ${ }^{5}$ and white-light slowing. ${ }^{6}$ There is growing interest ${ }^{7}$ in extending such slowing and cooling methods to molecules, especially polar molecules, whose rich internal structure and strong dipolar interactions make them candidates for quantum computing, ${ }^{8}$ cold chemistry, ${ }^{9}$ precision measurement, ${ }^{10-13}$ and observation of novel quantum phases. ${ }^{14}$ Laser cooling of a molecule has recently been demonstrated, ${ }^{15,16}$ and other existing techniques to produce cold and slow beams of polar molecules include using time-varying electric fields to decelerate a supersonic expansion, ${ }^{17}$ filtering slow molecules from a cold source, ${ }^{18}$ and buffer gas cooling ${ }^{19}$ of both pulsed ${ }^{20}$ and continuous ${ }^{21}$ beams.

In this paper we report on a newly developed buffer gas cooled beam source. We study ThO beams from both helium and neon buffer-gas-based beam sources, and observe cooling of ThO from the free expansion of the buffer gas. The flux, divergence, temperature, and velocity of the buffer gas beams we studied compare favorably (and for the case of chemically reactive molecules ${ }^{22}$ very favorably) to supersonic or effusive beams, as discussed further in Section 3.7. Buffer gas beams have been successfully utilized in the past to create cold and slow beams of several species, ${ }^{19,23,24}$ including (but not limited to) 

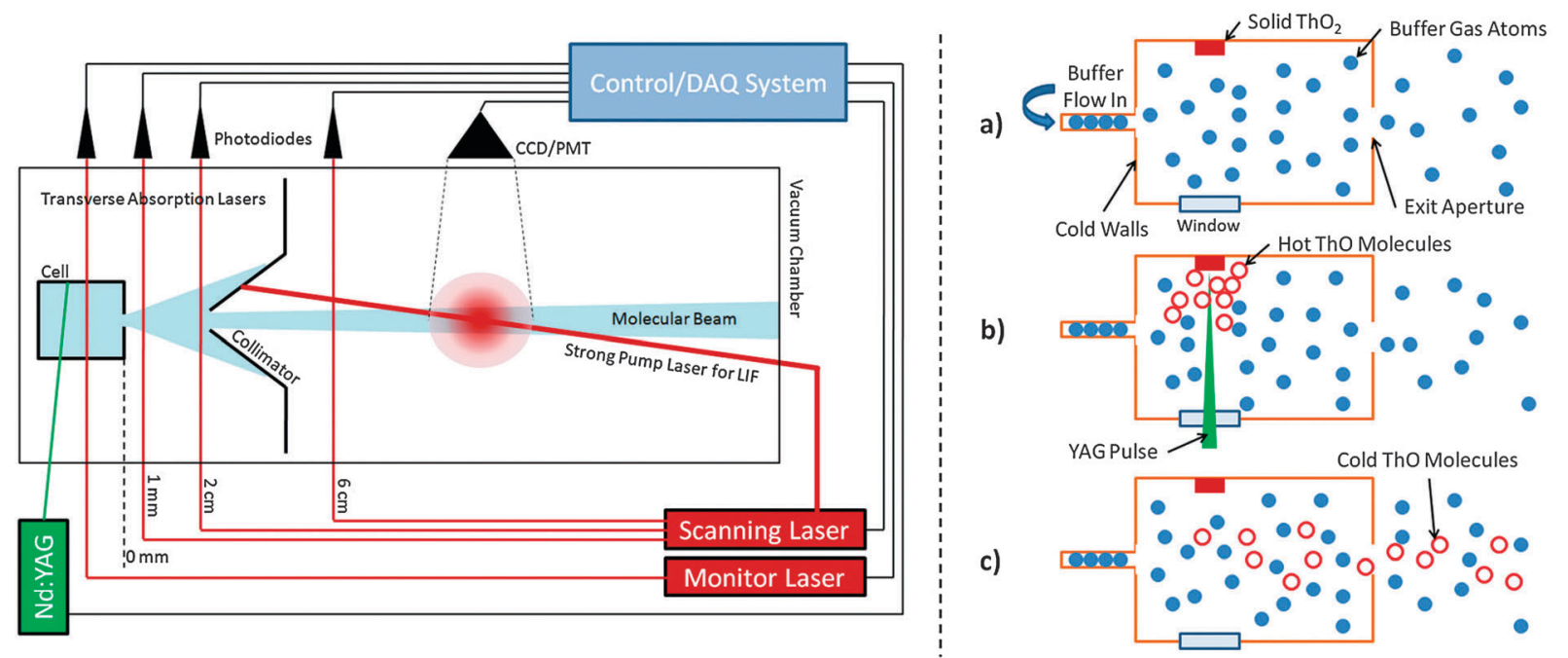

Fig. 1 Left: The cold beam apparatus. The features inside the vacuum chamber are described in Section 2, and the features outside the vacuum chamber are described in Section 3. Right: A detailed view of the cell. (a) A continuous gas flow maintains a stagnation density of buffer gas atoms (filled blue circles) in thermal equilibrium with a cold cell. A solid piece of $\mathrm{ThO}_{2}$ is mounted to the cell wall. (b) A YAG pulse vaporizes a portion of the target and ejects hot ThO molecules (empty red circles). (c) Collisions with the buffer gas cool the molecules, which then flow out of the cell to form a beam.

$\mathrm{ND}_{3}, \mathrm{O}_{2}, \mathrm{PbO}, \mathrm{SrF}, \mathrm{SrO}, \mathrm{ThO}, \mathrm{Na}, \mathrm{Rb}$, and $\mathrm{Yb}$. The beams presented in the current work operate at a much higher Reynolds number than previously demonstrated buffer gas beams, and we observe new and advantageous features, in particular expansion cooling, not seen in previous work (though similar results were observed concurrently by a collaborating group ${ }^{24}$ ).

Both helium and neon cooled beams were studied. Helium gas can be used at lower temperatures, and can therefore be used to lower the initial ThO temperature below that of neonbased sources. However, neon has much simpler pumping requirements to maintain good vacuum in the beam region, and a neon-based source operates at a higher temperature which allows for larger heat loads for molecule production. We find that due to expansion cooling in neon, the final, optimized beam temperatures for both helium- and neonbased beams are very similar. For a planned precision spectroscopy experiment with $\mathrm{ThO}^{10}$ the neon-based source is superior in certain aspects (particularly technical ones) to that of the helium-based source. Further comparison of heliumand neon-based sources can be found in Section 3.6.

\section{Apparatus}

The heart of our cold beam apparatus (see Fig. 1) is similar to that which is described in earlier buffer gas cooled beam publications, ${ }^{20,21,23}$ to which the reader is referred for additional technical details. It is a cryogenically cooled, cylindrical copper cell with internal dimensions of $13 \mathrm{~mm}$ diameter and $75 \mathrm{~mm}$ length. A $2 \mathrm{~mm}$ inner diameter tube entering on one end of the cylinder flows buffer gas into the cell. A $150 \mathrm{~mm}$ length of the fill line is thermally anchored to the cell, ensuring that the buffer gas is cold before it flows into the cell volume. An open aperture (or nozzle) on the other end of the cell lets the buffer gas spray out as a beam, as shown in Fig. 1. ThO molecules are injected into the cell via ablation of a ceramic target of $\mathrm{ThO}_{2}$, located approximately $50 \mathrm{~mm}$ from the exit aperture. A pulsed Nd:YAG $\operatorname{laser}^{25}$ is fired at the $\mathrm{ThO}_{2}$ target, creating an initially hot plume of gas-phase $\mathrm{ThO}$ molecules (along with other detritus of the ablation process). Hot ThO molecules mix with the buffer gas in the cell, and cool to near the cell temperature, typically between 4 and $20 \mathrm{~K}$. The buffer gas is flowed continuously through the cell at a rate $f_{0}=1-100 \mathrm{SCCM}+\dagger$ This both maintains a buffer gas stagnation density of $n_{0} \approx 10^{15}-10^{16} \mathrm{~cm}^{-3}\left(\sim 10^{-3}-10^{-2}\right.$ torr $)$ and extracts the molecules out of the aperture into a beam. ${ }^{21}$ The result, due to the pulsed introduction of ThO into the cell, is a pulsed beam of $\mathrm{ThO}$ molecules (embedded in a continuous flow of buffer gas) over a 1-3 ms period, as shown in Fig. 2. We have achieved stable operation of the neon-based beam with up to a $200 \mathrm{~Hz}$ repetition rate; however, the data presented in this paper are at a repetition rate of $10 \mathrm{~Hz}$. The cell aperture is a square hole of adjustable side length $d_{\mathrm{a}}=0-4.5 \mathrm{~mm}$ that can be varied in situ and continuously while the beam runs.

Either helium or neon is used as the buffer gas (typically called the carrier gas in most beam literature). The cell temperature $T_{0}$ is maintained at $5 \pm 1 \mathrm{~K}$ for helium, or $18 \pm 1 \mathrm{~K}$ for neon, and is controlled by the use of resistive heaters thermally anchored to the cell. The cell is surrounded by a radiation shield at $4 \mathrm{~K}$, which is partially covered in activated charcoal ${ }^{26}$ to form a cryopump that keeps the helium background pressure low. A second radiation shield at temperature $\sim 50 \mathrm{~K}$ surrounds the $4 \mathrm{~K}$ radiation shield, and both shields have glass windows to allow the transmission of spectroscopy lasers, and holes to allow passage of the molecular beam. The radiation shields and cell are connected by flexible copper braid heat links to a pulse tube refrigerator. ${ }^{27}$

$\dagger 1 \mathrm{SCCM}=1 \mathrm{~cm}^{3}$ per minute of gas at standard conditions, or about $4.5 \times 10^{17}$ gas atoms per second. 

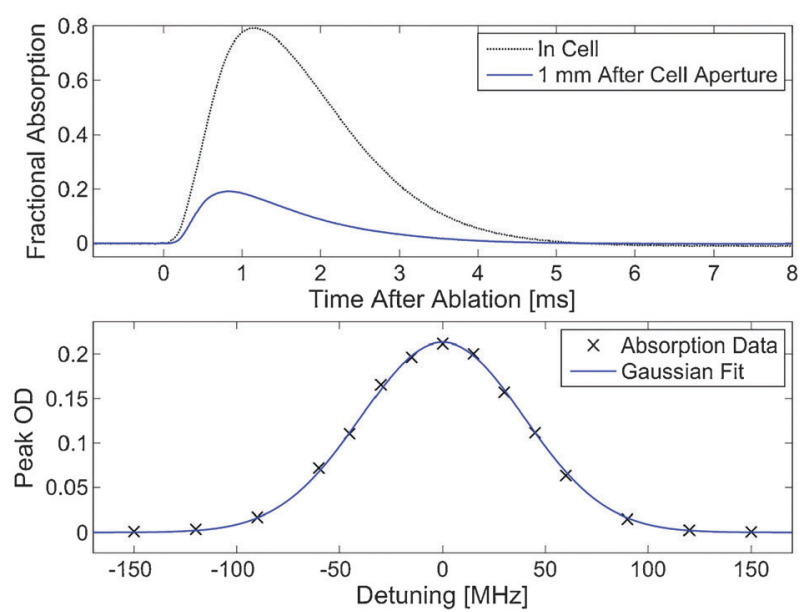

Fig. 2 Top: An absorption signal of the ThO molecular beam in a 1 SCCM flow of helium buffer gas with a $4.5 \mathrm{~mm}$ diameter cell aperture. The laser is fixed spatially, so the time variation of the signal is a result of the molecular beam pulse passing through the laser. The different traces show absorption in the cell (dotted) and $1 \mathrm{~mm}$ after the cell aperture (solid). For these data, the laser is locked on the resonance of the $\mathrm{X}-\mathrm{C} Q(1)$ line. The width and height of the signals depend on the buffer gas flow rate, buffer gas species, and cell aperture size; however, the qualitative features are similar. Bottom: By varying the laser detuning, we obtain an absorption spectrum. This curve shows the absorption spectrum of the molecular beam $1 \mathrm{~mm}$ after the cell, with the same experimental parameters as in the top figure. Optical density (OD) is defined as $T=e^{-\mathrm{OD}}$, where $T$ is the transmitted fraction of the probe laser light.

The beam exiting the cell is incident on a conical collimator with a $6 \mathrm{~mm}$ diameter orifice located $25 \mathrm{~mm}$ from the cell aperture. There are expected to be few collisions this far from the cell aperture, so for our work, the features of this collimator are more akin to those of a simple differentially pumped aperture. For helium buffer gas, the collimator is at a temperature of about $8 \mathrm{~K}$. For neon buffer gas the collimator is heated to $30 \mathrm{~K}$ to prevent neon ice formation.

All of the cryogenic apparatus is kept inside a roomtemperature vacuum chamber of internal volume $\sim 0.2 \mathrm{~m}^{3}$. During beam operation, vacuum is maintained by cryopumping of the charcoal and radiation shields, and no external pumping on the vacuum chamber is required. However, a small $\left(601 \mathrm{~s}^{-1}\right)$ turbomolecular pump continuously pumps on the chamber, to pump out any gas released should the cryopumps warm up and stop pumping. The main chamber has a pressure of $<10^{-5}$ torr during buffer gas flow, as measured with an ion gauge.

The $\mathrm{ThO}_{2}$ target is constructed from $\mathrm{ThO}_{2}$ powder, pressed and sintered as described in existing literature. ${ }^{28}$ These targets typically yield $>30000$ YAG shots on a single focus site before the yield per shot drops to $50 \%$ of the initial value, at which time the focus must be moved to a new spot. The large surface area $\left(\sim 1 \mathrm{~cm}^{2}\right)$ of the target should allow for $>10^{7}$ shots before target replacement is necessary.

\subsection{Qualitative features of buffer gas beams}

Here we present a brief overview of the features of a buffer gas cooled beam, which are discussed in detail in the existing literature. ${ }^{29}$ Central to understanding the unique properties of buffer gas cooled beams is the process of hydrodynamic entrainment. ${ }^{21}$ A "hydrodynamic" buffer gas cooled beam is designed so that the characteristic pumpout time ( $\tau_{\text {pump }}$ ) for the molecules to exit the cell is less than the characteristic diffusion time to the cell walls $\left(\tau_{\mathrm{diff}}\right),{ }^{30}$ both of which are typically $1-10 \mathrm{~ms}$. These conditions result in many of the molecules being extracted from the cell before they diffuse and stick to the cold cell walls. The ratio of these timescales is given by

$$
\gamma_{\text {cell }} \equiv \frac{\tau_{\text {diff }}}{\tau_{\text {pump }}} \approx \frac{f_{0} \sigma}{\bar{v}_{0, \mathrm{~b}} L_{\text {cell }}},
$$

where $\sigma$ is the cross section for collisions between ThO and the buffer gas, $\bar{v}_{0, \mathrm{~b}}$ is the mean thermal velocity of the buffer gas atoms (the subscript 0 indicates in-cell, stagnation quantities), and $L_{\text {cell }}$ is the length of the cell. The parameter $\gamma$ determines whether the beam system is running with hydrodynamic entrainment or not. When $\gamma_{\text {cell }} \gtrsim 1$ the molecules are entrained in the flow of the buffer gas, which results in an order of unity fraction of cooled, in-cell molecules being extracted into the molecular beam. For a hydrodynamic source, during the initial cooling and through the extraction phase, the molecules are in a low-density environment colliding only with inert gas atoms. These aspects of hydrodynamic buffer gas beams make this method an attractive alternative for species that are reactive, refractory, or otherwise difficult to obtain in the gas phase.

In the beam region just outside the cell aperture, the molecules undergo collisions with buffer gas atoms. Because the average velocity of the buffer gas atoms is higher than that of the (typically) heavier molecules, and because the collisions are primarily in the forward direction, the molecules are accelerated to a forward velocity, $v_{\mathrm{f}}$, which is larger than the thermal velocity of the molecules (just as with supersonic beams ${ }^{2}$ ). As the stagnation density is increased, $v_{\mathrm{f}}$ increases until it approaches a maximum value, which is that of the forward velocity of the buffer gas. In flow regimes considered in the existing literature, the transverse spread is given approximately by a Maxwell-Boltzmann distribution with temperature equal to the cell temperature, since there are typically not enough collisions outside the cell to increase this spread. In this model the molecular beam is therefore a thermal cloud of molecules with a center of mass motion given approximately by the forward velocity of the buffer gas. The angular distribution has a characteristic full (apex) angle $\theta$ given by $\tan (\theta / 2)=v_{\perp} / v_{\|} \approx \bar{v}_{0, \mathrm{~mol}} / \bar{v}_{0, \mathrm{~b}} \approx \sqrt{m_{\mathrm{b}} / m_{\mathrm{mol}}}$, where the subscripts $\mathrm{b}$ and mol represent the buffer gas and molecule, respectively. Since typically $m_{\mathrm{mol}} \gg m_{\mathrm{b}}$, we have $\theta \ll 1$ so $\theta \approx 2 \sqrt{m_{\mathrm{b}} / m_{\mathrm{mol}}}$. The characteristic solid angle is then $\Omega=2 \pi(1-\cos (\theta / 2)) \approx \pi \theta^{2} / 4=\pi m_{\mathrm{b}} / m_{\text {mol }}$. This quantity is approximately 0.1 and 0.3 for $\mathrm{ThO}$ in $\mathrm{He}$ and $\mathrm{Ne}$, respectively. This model accurately describes the results of previous buffer gas beam experiments. ${ }^{20,21,23}$ As will be seen in Section 3, we have studied beam operation at higher Reynolds numbers than previous experiments, and therefore additional effects must be considered.

2.1.1 Flow parameterization. Buffer gas beams typically operate in the intermediate regime between effusive and 
supersonic, where both molecular kinetics and fluid-like behavior are important. The parameters determining the operating regime of the beam dynamics outside the cell are the Reynolds and Knudsen numbers. The Knudsen number is defined as $K n=\lambda / d \approx\left(\sqrt{2} n_{0} \sigma d\right)^{-1}$, where $\lambda$ is the mean free path of the buffer gas and $d$ is a characteristic length scale, in our case the cell aperture diameter $d_{\mathrm{a}}$. The Knudsen number is related to the Mach number, $M a$, and Reynolds number, $R e$, by ${ }^{31}$

$$
\frac{1}{2}(K n)(R e) \approx M a
$$

Near the cell aperture, the buffer-gas atoms are traveling at approximately their thermal velocity $\bar{v}_{0, \mathrm{~b}}=\sqrt{8 k_{\mathrm{B}} T_{0} / \pi m_{\mathrm{b}}}$, where $k_{\mathrm{B}}$ is Boltzmann's constant. For the purposes of our qualitative estimates, this velocity is near to the speed of sound $c_{0, \mathrm{~b}}=\sqrt{5 k_{\mathrm{B}} T_{0} / 3 m_{\mathrm{b}}}=0.8 \bar{v}_{0, \mathrm{~b}}$. Therefore $M a \approx 1$ near the cell aperture, and we have the relation

$$
\frac{1}{2}(K n)(R e) \approx 1
$$

Using the formulas above, we estimate the relationship between flow and Reynolds number to be

$$
R e \approx 0.7 \times\left(f_{0} / 1 \mathrm{SCCM}\right) \times\left(d_{\mathrm{a}} / 4.5 \mathrm{~mm}\right),
$$

where we estimate the collision cross section (from diffusion measurements) to be $\sigma \approx 3 \times 10^{-15} \mathrm{~cm}^{2}$.

Buffer gas beams typically operate ${ }^{19}$ in the regime $K n \approx$ $1-10^{-2}$. Effusive beams require $K n>1$ so that the aperture does not alter the properties of the atoms extracted from the cell, while supersonic beams typically ${ }^{2}$ have $K n \lesssim 10^{-3}$.

\section{Measured beam properties}

We studied the beam with a variety of buffer gas flows, aperture sizes, and cell temperatures for both helium and neon buffer gases using continuous wave laser spectroscopy from the ThO ground electronic state $\mathrm{X}\left(v=0, \Omega=0^{+}\right.$, $\left.B_{\mathrm{e}}=0.33 \mathrm{~cm}^{-1}\right)$ to the excited electronic state $\mathrm{C}(v=0$, $\left.\Omega=1, T_{0}=14489.90 \mathrm{~cm}^{-1}, B_{\mathrm{e}}=0.32 \mathrm{~cm}^{-1}\right)^{32}$ at $690 \mathrm{~nm}$. Two diode lasers are each locked to a stabilized frequency source via a Fabry-Pérot transfer cavity. The frequency of one laser is scanned to obtain spectra, while the other is kept at a fixed frequency and used to normalize against variation in the ablation yield (typically a few percent from shot-to-shot). The scanning laser detuning, Nd:YAG pulses, and data acquisition are all synchronized via a master control computer. The scanning laser is split into multiple beams and used for absorption transverse to the molecular beam at several distances after the cell aperture, and for laser-induced fluorescence (LIF) parallel to the molecular beam as shown in Fig. 1. Absorption data are obtained using silicon photodiodes, and laser-induced fluorescence is collected with either a CCD camera or a photomultiplier tube (PMT).

\subsection{Rotational cooling}

The rotational temperature was determined by fitting a Boltzmann distribution to the lowest six rotational levels ( $J=0$ to $J=5$ ) of the ground state X. Population was determined from the optical density (see Fig. 2) of absorption on the $\mathrm{X}$ to $\mathrm{C}$ transition. The lines $R(0), Q(1), \ldots, Q(5)$ were used to obtain the population in $\mathrm{X}, J=0,1, \ldots, 5$ respectively. Rotational temperatures with both helium and neon buffer gases are shown in Fig. 3. The minimum measured rotational temperature, measured $6 \mathrm{~cm}$ from the cell, as a function of buffer gas flow and aperture size, was $2.0 \pm 0.8 \mathrm{~K}$ with neon buffer gas, and $1.7 \pm 0.5 \mathrm{~K}$ with helium buffer gas (error bars in this paper refer to the $95 \%$ confidence interval of fits). These represent an increase of a factor of 8.2 and 2.8 in the $\mathrm{X}, J=0$ population with neon and helium buffer gases, respectively, from the distribution present at the cell temperature.

With neon buffer gas the rotational temperature decreases with both increasing flow and increasing distance from the cell aperture. The rotational temperature does not change after a distance of $2 \mathrm{~cm}$ after the cell, indicating that the cooling collisions have stopped before this distance. With helium buffer gas the rotational temperature is largely independent of flow, distance from the cell, and aperture size as measured with 14 different flows, three different distances after the cell, and three different aperture sizes. The temperature of the molecules just outside of the cell, however, is lower than the cell temperature, even for the lowest flow and largest aperture. This behavior is unexpected: At the lowest flow (1 SCCM) and the largest aperture ( $4.5 \mathrm{~mm}$ side square), the flow regime is effusive $(K n \approx 1)$ and so we should see no additional cooling below the cell temperature of $5 \mathrm{~K}$. More low-flow helium phenomena, along with possible explanations, are discussed in Section 3.5.2.

For both buffer gases, the molecules approach some minimum rotational temperature that does not decrease with additional flow. This minimum temperature appears to be about $2-3 \mathrm{~K}$, and is similar for both helium and neon. In fact, the rotational temperature just outside a $9 \mathrm{~K}$ cell with a helium buffer gas flow has a similar minimum temperature of $2.9 \pm 1.3 \mathrm{~K}$.

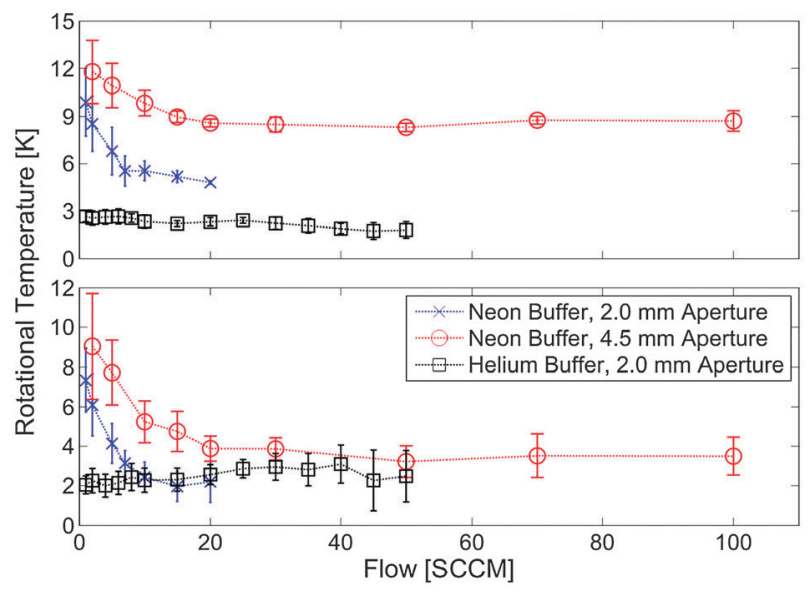

Fig. 3 Rotational temperatures in the buffer gas cooled beam. Top: Temperatures measured $1 \mathrm{~mm}$ from the cell aperture. Bottom: Final rotational temperatures in the expansion, measured $6 \mathrm{~cm}$ after the cell aperture. The cell temperatures are $5 \pm 1 \mathrm{~K}$ and $18 \pm 1 \mathrm{~K}$ for helium and neon buffer gases, respectively. The data with a helium buffer gas were taken with a $2.0 \mathrm{~mm}$ aperture, while the data with a neon buffer gas were taken with both $2.0 \mathrm{~mm}$ and $6.5 \mathrm{~mm}$ apertures. 


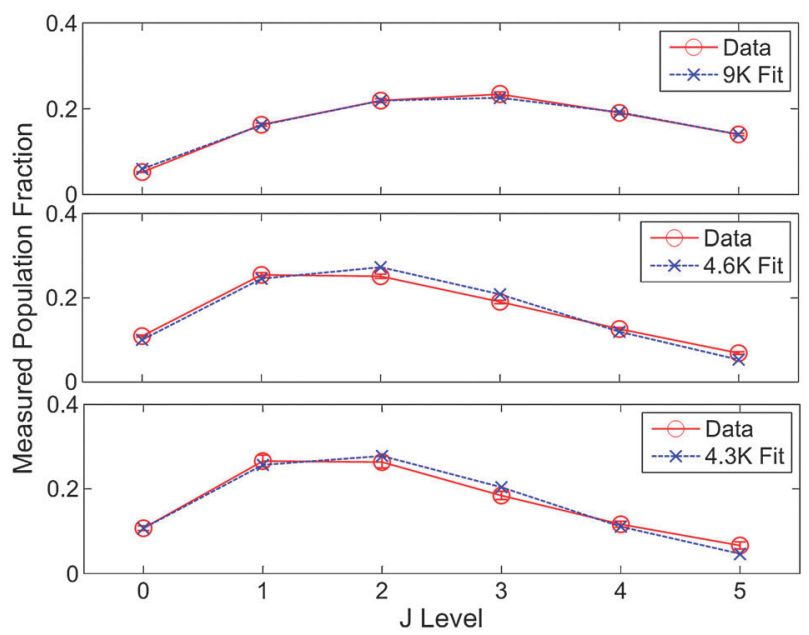

Fig. 4 Rotational level populations with Boltzmann distribution fits for $30 \mathrm{SCCM}$ neon flow, $4.5 \mathrm{~mm}$ aperture. The top, middle, and bottom plots show the distributions $1 \mathrm{~mm}, 2 \mathrm{~cm}$, and $6 \mathrm{~cm}$ from the cell aperture, respectively.

\subsection{Forward velocity}

The forward velocity of the ThO beam was measured at distances between about $6 \mathrm{~cm}$ and $16 \mathrm{~cm}$ from the cell aperture using laser-induced fluorescence (LIF) imaging. A counterpropagating, red-detuned pump beam excites the molecules on the $Q(1)$ or $Q(2)$ line of the $\mathrm{X}-\mathrm{C}$ transition, and the fluorescence is collected with either a CCD camera or a PMT. The camera gives spatial information about the beam, but since the exposure time is longer than the molecular pulse duration the camera averages over an entire pulse. To get time-dependent information, we use the PMT. We measure the first-order Doppler shift of the molecules by fitting a gaussian shape to the obtained LIF spectrum and comparing the center to that of a transverse absorption spectrum. The forward velocity and velocity distribution are then inferred from the first-order Doppler shift and width of the spectrum, respectively.

Values for the forward velocity are between 120 and $200 \mathrm{~m} \mathrm{~s}^{-1}$, which are slower than typical supersonic expansions. The final velocity of a supersonic expansion of a monoatomic carrier gas is given by ${ }^{33}$

$$
v_{\|}^{*}=\sqrt{\frac{52 \frac{2 k_{\mathrm{B}} T_{0}}{2}}{m}} \approx 1.6 v_{\mathrm{p}, 0},
$$

where $m$ is the carrier gas particle mass, $T_{0}$ is the stagnation temperature in the source, and $v_{\mathrm{p}, 0}$ is the most probable thermal velocity of the carrier in the source. From this equation, the final velocity is about $600 \mathrm{~m} \mathrm{~s}^{-1}$ for a supersonic expansion of room-temperature argon, or about $300 \mathrm{~m} \mathrm{~s}^{-1}$ for an expansion of $210 \mathrm{~K}$ xenon. With neon buffer gas in an $18 \mathrm{~K}$ cell, the forward velocity (see Fig. 5) approaches a value that is very close to the final velocity of $\sim 200 \mathrm{~m} \mathrm{~s}^{-1}$ predicted by eqn (4). With helium buffer gas, the mean forward velocity approaches about $70 \%$ of the value $230 \mathrm{~m} \mathrm{~s}^{-1}$ predicted by eqn (4) for a $5 \mathrm{~K}$ cell. The lower-than-expected value for the helium-cooled beam is likely due to collisions with the background helium that accumulates due to the limited pumping speed of the activated charcoal, which is not a problem with
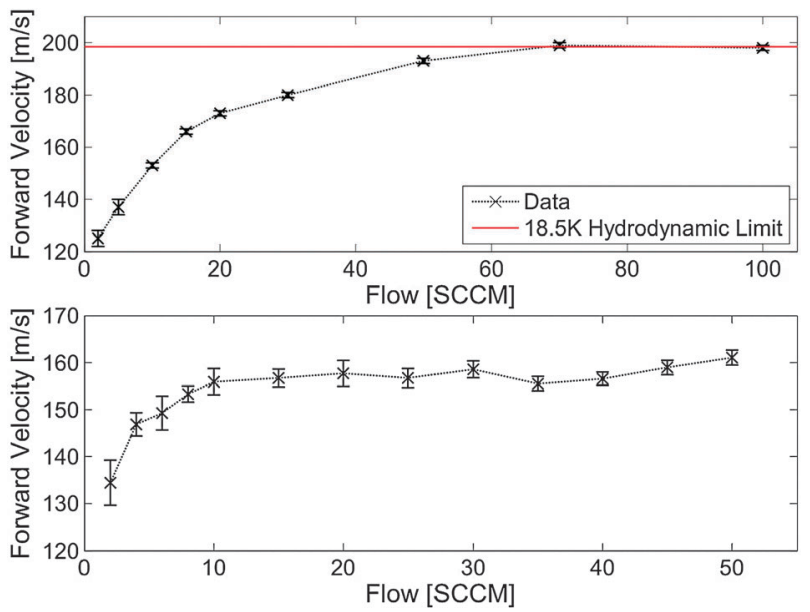

Fig. 5 Top: Mean forward velocity $v$ s. flow rate with neon buffer gas. The solid line is the hydrodynamic limit for the forward velocity of neon atoms exiting an $18.5 \mathrm{~K}$ cell, given by eqn (4). The forward velocity of the molecules with neon buffer gas cooling varies by no more than $10 \%$ over a pulse. Bottom: Mean forward velocity $v$ s. flow rate with helium buffer gas.

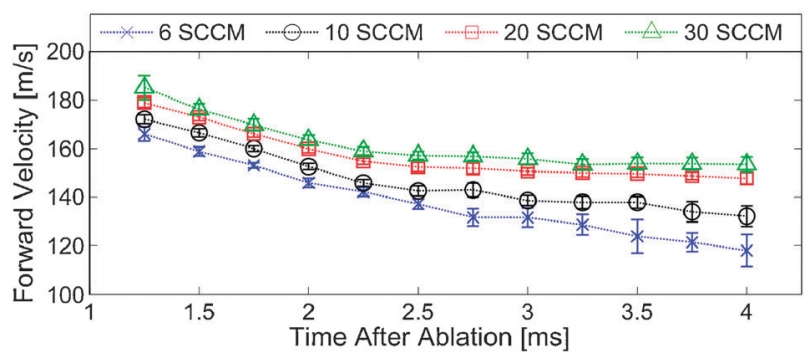

Fig. 6 Forward velocity $v$ s. time after ablation for several different flow rates of the helium buffer gas.

neon because once it sticks to a $4 \mathrm{~K}$ surface, it remains there nearly indefinitely (i.e. has negligible vapor pressure at $4 \mathrm{~K}$ ). Increasing the amount of charcoal and improving its placement mitigated this problem; however, the issue remained present. Further discussion is presented in Section 3.6.

Interpretation of the helium forward velocity data is additionally complicated by the fact that, unlike with the case of neon buffer gas, it varies in time over the molecule pulse duration, as shown in Fig. 6. This dependence of beam properties on time after ablation is perhaps due to the finite amount of time required to thermalize the ThO molecules in the buffer gas cell, which is much smaller with neon due to neon's smaller mass mismatch with $\mathrm{ThO}$, and the fact that the heat introduced by ablation results in a smaller fractional change in temperature at $18 \mathrm{~K}$ versus $5 \mathrm{~K}$. Additionally, the forward velocity with helium buffer gas varies by as much as $\sim 10 \%$ if the ablation spot is moved, and as the charcoal cryopumps become full of helium and the pumping speed changes, as discussed in Section 3.6. These effects are not observed with neon buffer gas.

3.2.1 The velocity vs. flow relationship. We can model the shape of the velocity vs. flow curve with the "sudden freeze" model, ${ }^{33}$ in which we assume that the molecules are in 
equilibrium with the buffer gas until some distance where the density is low enough that the gases decouple, and the molecular beam properties are frozen. The velocity of the buffer gas scales with the density $n$ as

$$
v(x) \approx 1.6 v_{\mathrm{p}, 0}\left[1-\left(\frac{n(x)}{n_{0}}\right)^{2 / 3}\right]^{1 / 2},
$$

where $v_{\mathrm{p}, 0}=\left(2 k_{\mathrm{B}} T_{0} / m_{\mathrm{b}}\right)^{1 / 2}$ is the most probable velocity of the buffer gas in the cell $\left(\sim 120 \mathrm{~m} \mathrm{~s}^{-1}\right.$ for $\left.17 \mathrm{~K} \mathrm{Ne}\right), k_{\mathrm{B}}$ is Boltzmann's constant, $x$ is the distance from the cell aperture, $n_{0}$ is the cell stagnation density, and the collision cross section is estimated to be $\sigma \approx 3 \times 10^{-15} \mathrm{~cm}^{2}$. Introducing the normalized distance $\xi \equiv x / d_{\mathrm{a}}$, the far-field number density scales as $n(\xi) / n_{0} \approx 0.2 \xi^{-2}$, so $v(\xi) \approx 1.6 v_{\mathrm{p}, 0} \sqrt{1-0.3 \xi^{-4 / 3}}$. For a monoatomic hard-sphere gas, the location where collisions freeze is given by

$$
\xi_{0} \approx\left(0.1 \sigma n_{0} d_{\mathrm{a}}\right)^{3 / 5} \approx 0.2\left(\frac{f_{0}}{1 \mathrm{SCCM}}\right)^{3 / 5}
$$

where in the last equality we used $d_{\mathrm{a}}=4.5 \mathrm{~mm}$. The $3 / 5$ exponent is model dependent and should be considered approximate. Therefore, if we assume that the molecules and buffer gas are in equilibrium until the position $\xi_{0}$, the final molecule velocity will be given by

$$
v\left(\xi_{0}\right) \approx 1.6 v_{\mathrm{p}, 0}\left[1-3\left(\frac{f_{0}}{1 \mathrm{SCCM}}\right)^{-4 / 5}\right]^{1 / 2}
$$

This sudden-freeze model is valid when there are collisions in the beam, i.e. when $\xi_{0} \gtrsim 1$, which from eqn (5) occurs at approximately $15 \mathrm{SCCM}$ neon flow with the $4.5 \mathrm{~mm}$ aperture. Below this flow we are in the regime where the forward velocity increases linearly, ${ }^{20}$ so we can fit a line to the flows $\leq 15$ SCCM and the sudden freeze model for flows $\geq 15$ SCCM, as shown in Fig. 7.

To estimate the slope of the linear part, we use the model from the earlier buffer gas beam literature, ${ }^{20}$ near the aperture, the molecules undergo approximately $d_{\mathrm{a}} / \lambda_{0} \approx \operatorname{Re} / 2$ collisions (from eqn (3)). Each of these collisions gives the molecules a momentum kick in the forward direction of about $\delta p_{\mathrm{mol}} \approx m_{\mathrm{b}} v_{\mathrm{b}}$,

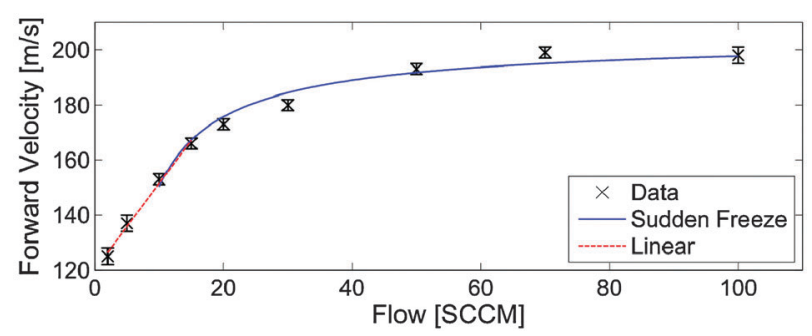

Fig. 7 Forward velocity for neon buffer gas with a $4.5 \mathrm{~mm}$ aperture, along with a linear fit at low flow and a sudden freeze fit at high flow. The sudden freeze fit is to the function $v=\alpha \sqrt{1-\beta f^{-4 / 5}}$, with parameters $\alpha=205 \pm 6 \mathrm{~m} \mathrm{~s}^{-1}, \beta=3.0 \pm 0.5$. The parameters are close to the expected values of $\alpha \approx 200 \mathrm{~m} \mathrm{~s}^{-1}$ (the final velocity) from experiment, and $\beta \approx 3$ from the estimate above. The linear model has a slope of $3.1 \pm 0.8\left(\mathrm{~m} \mathrm{~s}^{-1}\right)$ per SCCM, in good agreement with our estimate of $4.5\left(\mathrm{~m} \mathrm{~s}^{-1}\right)$ per SCCM. so the net velocity boost is $\Delta v_{\mathrm{mol}} \approx\left(d_{\mathrm{a}} / \lambda_{0}\right) \delta p_{\mathrm{mol}} \approx$ $(R e) v_{\mathrm{b}} m_{\mathrm{b}} / 2 m_{\mathrm{mol}}$. The forward velocity of the buffer atoms is $1.3 v_{\mathrm{p}, 0} \approx 160 \mathrm{~m} \mathrm{~s}^{-1}$, which is the mean forward velocity of an effusive beam ${ }^{2}$ ). The estimated conversion from flow to Reynolds number is given by $R e \approx 0.7 \times\left(f_{0} / 1 \mathrm{SCCM}\right) \times$ $\left(d_{\mathrm{a}} / 4.5 \mathrm{~mm}\right)$, so the velocity increase is estimated to be approximately given by $\Delta v_{\mathrm{mol}} \approx 4.5 \times f_{0} /(1 \mathrm{SCCM})$. A fit with this model is shown in Fig. 7, where we find good agreement with our estimated parameters.

\subsection{Divergence}

The width of the transverse absorption spectra is due to Doppler broadening, and therefore can be used to obtain the transverse velocity spread of the molecular beam. We divide the full width at half maximum (FWHM) of the transverse velocity distribution (at a distance of $2 \mathrm{~cm}$ from the cell aperture) by the forward velocity to obtain $\theta_{\text {FWHM }}$, the FWHM of the angular distribution. The angle $\theta_{\mathrm{FWHM}}$ is related to the half-maximum solid angle $\Omega$ by $\Omega=2 \pi\left(1-\cos \left(\theta_{\mathrm{FWHM}} / 2\right)\right)$. Though the molecular beam collimator is located only $5 \mathrm{~mm}$ from where the transverse velocity spread is measured (at a distance of $25 \mathrm{~mm}$ from the cell aperture), changing the temperature of the beam collimator does not alter the measured spectra, from which we conclude that the presence of the collimator does not significantly perturb the molecular beam. This conclusion is in agreement with the assumption, based on estimates of beam density near the collimator, that there are few collisions near the collimator.

For neon buffer gas, the divergence has a minimum at $\Omega \approx 0.35 \pm 0.03\left(\theta_{\mathrm{FWHM}} \approx 39^{\circ}\right)$, close to the hydrodynamic entrainment prediction of $\pi m_{\mathrm{Ne}} / m_{\mathrm{ThO}} \approx 0.3$. The location of the minimum divergence appears at approximately the same Reynolds number for different apertures; however, as discussed in Section 3.5.1, the neon signals at smaller aperture are greatly reduced, and therefore the minimum cannot be determined as accurately.

For helium buffer gas, the minimum divergence of about $\Omega \approx 0.22 \pm 0.06\left(\theta_{\mathrm{FWHM}} \approx 30^{\circ}\right)$ occurs for 2 SCCM flow, and then steadily increases to $0.29 \pm 0.07$ at 50 SCCM. The smallest measured divergence was $\Omega \approx 0.17 \pm 0.06$ $\left(\theta_{\mathrm{FWHM}} \approx 27^{\circ}\right)$ with a $3.0 \mathrm{~mm}$ aperture; however, changing the aperture size does not have an appreciable effect on the divergence $v s$. flow relationship.

The spectra analyzed in this section were obtained by integrating the optical density of the molecular beam pulse vs. time after the YAG pulse and varying the absorption laser detuning (see Fig. 2). Integrating over the entire pulse yields the mean transverse spread of all the molecules. Alternatively, we can look at the transverse width as a function of time after the ablation pulse. With neon buffer gas the transverse spread does not change by more than $10 \%$ over the duration of a single pulse of molecules, but with helium the transverse spread changes by as much as $30 \%$. The divergence shown in Fig. 8 was calculated using the mean spectral width, defined as the spectral width of the entire molecule pulse. As with the forward velocity data, the transverse velocity spread with helium buffer gas varies by as much as $\sim 10 \%$ if the ablation spot is moved, and as the charcoal cryopumps become full of 


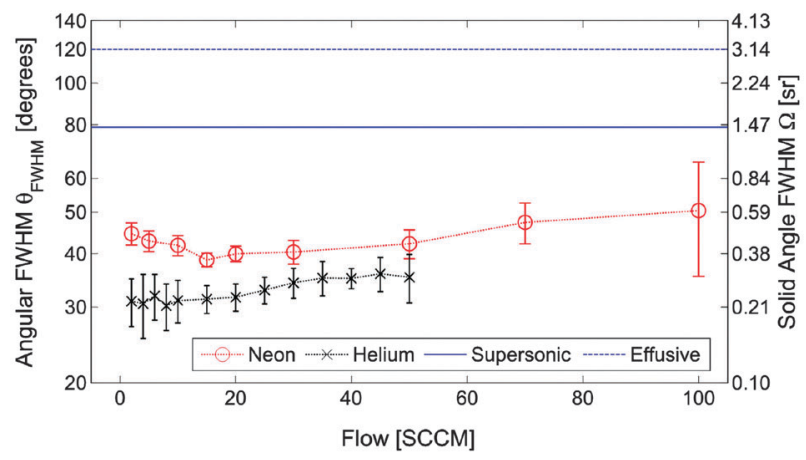

Fig. 8 Divergence in the beam with a $4.5 \mathrm{~mm}$ aperture, measured $2 \mathrm{~cm}$ after the cell aperture, compared to the divergences of supersonic and effusive beams. ${ }^{2}$ The cell temperatures are $18 \pm 1 \mathrm{~K}$ for neon, $5 \pm 1 \mathrm{~K}$ for helium. For this aperture size, the conversion from flow to Reynolds number is $R e \approx 0.7 \times$ flow $/(1 \mathrm{SCCM})$.

helium; however, the data typically fall within the error bars shown in Fig. 8. These effects are not observed with neon buffer gas.

3.3.1 The divergence vs. flow relationship. Here we present a model to describe how the beam divergence varies with flow, similar to those discussed elsewhere. ${ }^{34,35}$ For low flows we are in the regime where forward-peaked collisions with the buffer gas near the aperture result in a forward velocity proportional to flow, but the transverse spread is fixed, as described in Section 2.1. The result is a linearly decreasing divergence with increasing flow. For high flows, the molecules are fully boosted to the forward velocity of the buffer gas. Additionally, there are an increasing number of collisions in the beam region between the ThO molecules and the buffer gas. Since the thermal distribution of the buffer gas defines its transverse velocity spread, which is larger than that of the molecules by a factor of $\sqrt{m_{\mathrm{mol}} / m_{\mathrm{b}}}$, collisions with the buffer gas in the beam increase the transverse velocity spread and therefore increase the divergence of the molecules. In this regime, the molecules outside of the aperture no longer constitute a thermal cloud as they do in the low flow case. Absorption spectra from lasers perpendicular to the molecular beam no longer give temperature information, but instead give the angular spread of the beam profile.

With a simple model, we can estimate the slope of the angular spread vs. flow curve. Near the aperture, the buffer gas atoms must follow a convergent trajectory since the flow cross-section narrows from the cell diameter $d_{\text {cell }} \approx 13 \mathrm{~mm}$ to the aperture diameter $d_{\mathrm{a}} \approx 5 \mathrm{~mm}$. The typical mean buffer gas velocity normal to the beam axis is given approximately by the flow velocity. The relationship between the in-cell flow velocity $v_{\text {cell }}$ and the flow velocity in the aperture, which is approximated by $v_{\mathrm{p}, 0}$, is given by $d_{\text {cell }}^{2} v_{\text {cell }} \approx d_{\mathrm{a}}^{2} v_{\mathrm{p}, 0}$, or $v_{\text {cell }} \approx$ $v_{\mathrm{p}, 0}\left(d_{\text {cell }} / d_{\mathrm{a}}\right)^{2} \approx 18 \mathrm{~m} \mathrm{~s}^{-1}$. There are $\sim \operatorname{Re} / 2$ collisions near the aperture, so the change in the transverse spread is given by $\Delta v_{\mathrm{mol}, \perp} \approx(R e / 2) v_{\mathrm{cell}} m_{\mathrm{b}} / m_{\mathrm{mol}} \approx\left(0.7 \mathrm{~m} \mathrm{~s}^{-1}\right) \times R e$, in good agreement with the typical data fit value of $\left(0.4 \mathrm{~m} \mathrm{~s}^{-1}\right) \times R e$, measured for several aperture sizes. The transverse spread downstream displays similar linear increases; however, modeling is complicated by the expansion dynamics. Typical slopes

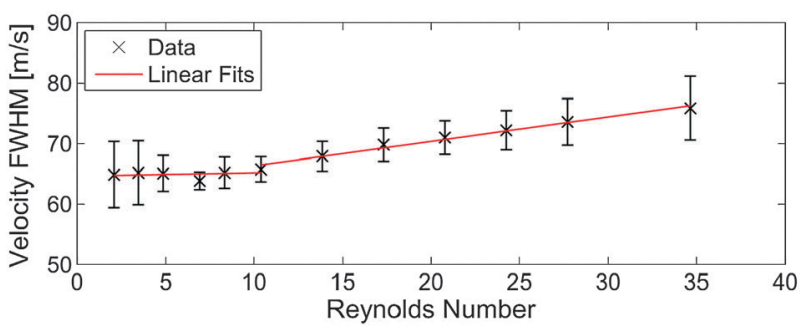

Fig. 9 The transverse spectral width $v s$. Reynolds number with neon buffer gas and a $4.5 \mathrm{~mm}$ cell aperture, measured at the aperture. At low flows the transverse widths inside the cell and outside the cell are the same, and indicate a thermal distribution of $T \approx 20 \mathrm{~K}$. The slope of the fit line at these low flows is $(0.06 \pm 0.27) \mathrm{m} \mathrm{s}^{-1}$, consistent with zero. At higher flows the transverse width in the beam begins to linearly increase, with slope $(0.41 \pm 0.06) \mathrm{m} \mathrm{s}^{-1}$. Note that the transition Reynolds number agrees with that from Fig. 7. For this aperture size, the conversion from flow to Reynolds number is $R e \approx 0.7 \times$ flow $/(1 \mathrm{SCCM})$.

for the transverse spread $v s$. Reynolds number relationship are $\sim 1\left(\mathrm{~m} \mathrm{~s}^{-1}\right) /(R e)$ (Fig. 9).

\subsection{Translational cooling}

The translational temperature in the longitudinal direction was measured by collecting laser-induced fluorescence spectra after excitation from a laser beam counter-propagating with respect to the molecular beam, similar to the procedure described in Section 3.2. The width of the spectrum is a result of Doppler broadening, so translational temperature is calculated by fitting the obtained spectrum to a Gaussian distribution and extracting the width. With neon buffer gas, the lowest translational temperature (in the center of mass frame of the molecular pulse) was $4.4 \pm 1.2 \mathrm{~K}$, corresponding to a velocity spread of $29 \pm 4 \mathrm{~m} \mathrm{~s}^{-1}$. Increasing the aperture diameter increases the final velocity spread, as shown in Fig. 10.

With helium buffer gas the data interpretation is more difficult because the spectral properties of the beam change as a function of time after ablation. Similar to the analysis for the helium forward velocity in Section 3.2, we can measure the time-resolved fluorescence from the molecules to extract spectral properties as a function of time after ablation. Fig. 11 shows the forward velocity spread for the entire molecule pulse, and the mean instantaneous forward velocity spread extracted from the time-resolved spectra. The instantaneous velocity spread is comparable to that with neon; however, the changing forward velocity of the beam (see Fig. 6) results in the forward velocity spread of the entire pulse being much larger.

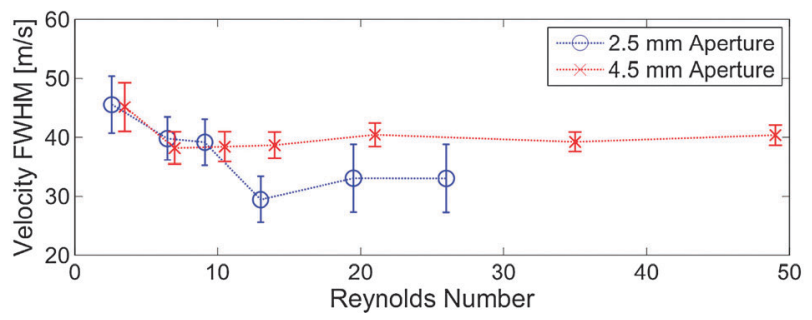

Fig. 10 The velocity spread in the forward direction with neon buffer gas. 


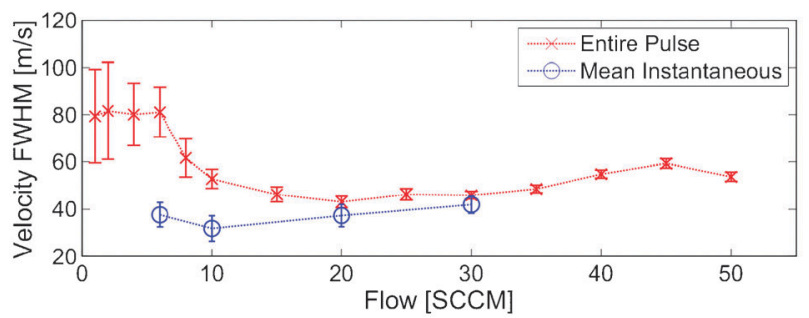

Fig. 11 The velocity spread in the forward direction with helium buffer gas. The data points marked by the $\times$ symbols indicate velocity spread averaged over the entire molecule pulse, while the data points marked by the $\bigcirc$ symbols indicate the mean instantaneous velocity spread obtained from time-resolved fluorescence data.

With neon, neither the forward velocity nor the forward velocity width change by more than $10 \%$ over the duration of the pulse.

\subsection{Cell extraction and molecule production}

We varied the flow and the aperture size with a fixed cell geometry and measured the molecule output from the cell. When the flow is large enough to ensure good extraction from the cell $\left(i . e . \gamma_{\text {cell }} \gtrsim 1\right)$, typical molecule outputs are $\sim 10^{11}$ in the $\mathrm{X}, J=0$ absolute ground state, or $\sim 10^{12}$ total molecules (in all states) per pulse, estimated from absorption spectra taken immediately after the cell aperture. The optical absorption cross section is estimated from the $\mathrm{C}$ state radiative decay lifetime and the calculated ${ }^{36}$ Franck-Condon factors for the transition. A lower bound of $493 \pm 38$ ns for the $\mathrm{C}$ state lifetime was measured by exciting molecules into the $\mathrm{C}$ state with resonant pump light, and then measuring the fluorescence as the molecules decay back into $\mathrm{X}$ after rapidly extinguishing the pump light. ${ }^{37}$ Since the temporal width of the pulse is $\sim 1 \mathrm{~ms}$, the peak instantaneous output rate is $\sim 10^{14}$ molecules per second per state in $J=0$, or $\sim 10^{15}$ total number of molecules per second. Behavior of the number of molecules output per pulse is plotted in Fig. 12. With neon buffer gas, the flow-output behavior is very similar for different aperture sizes larger than about $3.0 \mathrm{~mm}$, and has a maximum output around $\gamma_{\text {cell }} \approx 1-2$. With helium buffer gas, the shape of the curve is non-repeatable; specifically, the shape changes if the YAG ablation spot is moved, which is not the case with neon. However, the approximate number output is typically within a factor of two of the data in Fig. 12 for all conditions.

According to the simple hydrodynamic entrainment theory, the extraction of molecules from the cell is governed by the parameter $\gamma_{\text {cell }}$ from eqn (1). This parameter does not have an explicit dependence on the aperture size; however, we find that there can be a strong dependence on the aperture size that varies with the gas type. For neon buffer gas, the extraction fraction is constant for cell apertures larger than about $3 \mathrm{~mm}$, then falls off rapidly with decreasing aperture size. For helium buffer gas, the maximum extraction fraction does not depend significantly on the aperture size. It should be noted that based on previous observations, both published ${ }^{20,21}$ and unpublished, the cell extraction is typically dependent, sometimes in puzzling ways, on species, ablation properties, internal cell geometry, and collimation geometry.
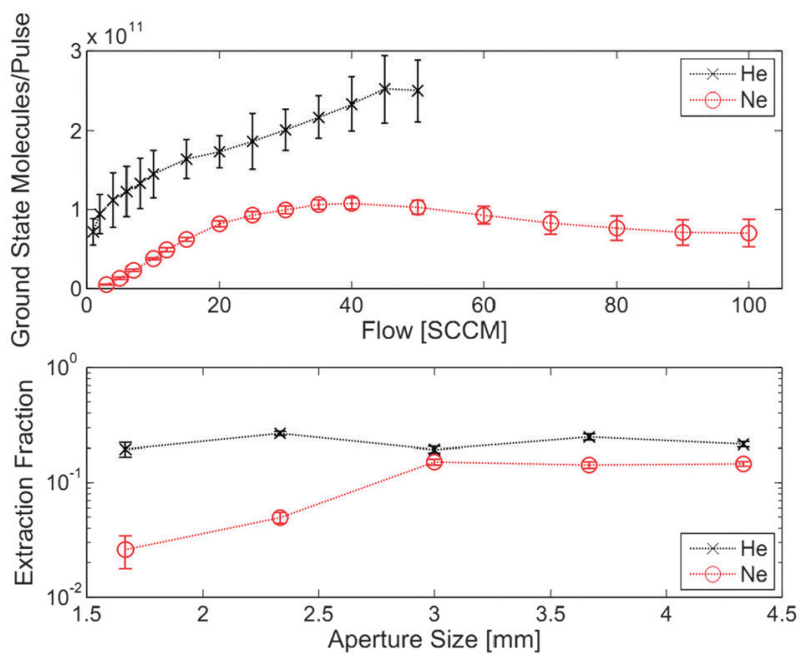

Fig. 12 Top: Number of absolute ground state molecules output per pulse with helium and neon buffer gases, using a $4.5 \mathrm{~mm}$ cell aperture. Bottom: Fraction of molecules extracted out of the cell into the beam, with neon and helium buffer gases.

3.5.1 High-flow neon phenomena. For very high neon flows (Reynolds number $\gtrsim 50$ ) the beam behavior degrades. The molecule pulse resulting from a single ablation shot begins to decompose into spatially and temporally variegated pulses with different spectral characteristics. This is likely a result of the post-ablation dynamics in the high density buffer gas inside the cell, as we have observed the onset of similar behavior occurring in high-density, ablation-loaded buffer gas cells in other species ${ }^{38}$ in the past. Fortunately, this behavior occurs for flows significantly above the point where the cell extraction is maximum, and therefore is not near the optimal flow for most conceivable beam-based spectroscopy experiments.

3.5.2 Low-flow helium phenomena. For low flows of helium the behavior of the beam is unusual and not in good agreement with other helium buffer gas beam experience. ${ }^{20}$ At low flow there should be little extraction and poor thermalization; however, in the data presented here we have evidence of a high-extraction, well-thermalized beam at flows as low as $1 \mathrm{SCCM}$ with the aperture size as large as $4.5 \mathrm{~mm}$, or $R e \approx 1$ and $\gamma_{\text {cell }}<0.1$. At this flow the mean free path in the cell is a few $\mathrm{mm}$, so it is surprising that there is any significant thermalization or extraction. A possible explanation for this behavior is that a helium film builds up in the cell (either on the walls or in the target) as helium gas flows into the cell between ablation pulses. Then, when the ablation pulse hits and the helium desorbs due to ablation heating, there is a pulse of higher buffer gas pressure at the moment the $\mathrm{ThO}$ is introduced into the cell. Additionally, we observe that the phenomena disappear as the cell is heated, in which case we observe the expected ${ }^{20}$ increasing extraction with increasing flow.

\subsection{Comparison of helium and neon buffer gas sources}

Our data show that a beam using neon buffer gas performs nearly as well as one using helium, but with much simpler technical requirements and a much larger cryopumping speed. 
Table 1 Comparison of the buffer gas beam sources presented in this paper to a supersonic source and an effusive source. Peak brightness is defined as the peak number of absolute ground state molecules per steradian per pulse (or per second for the continuous effusive source) in the beam. The buffer gas sources are those presented in this paper, and the range of values given for the beam properties is the range of values measured with varying gas flow rates and aperture sizes. The supersonic beam is a pulsed beam of YbF seeded in Xe from a $193 \mathrm{~K}$ source, ${ }^{22}$ and the effusive beam is an estimate for a $2000 \mathrm{~K}$ source based on ThO vapor pressure data ${ }^{39}$

\begin{tabular}{|c|c|c|c|c|}
\hline Beam type & Peak brightness & Velocity $/ \mathrm{m} \mathrm{s}^{-1}$ & $T_{\text {rotational }} / \mathrm{K}$ & $T_{\text {translational }} / \mathrm{K}$ \\
\hline Ne buffer gas (ThO, this work) & $3 \times 10^{11}$ per sr per pulse & $120-200$ & $2-9$ & $4-11$ \\
\hline He buffer gas (ThO, this work) & $10 \times 10^{11}$ per sr per pulse & $120-160$ & $2-3$ & $5-10^{a}$ \\
\hline Supersonic $\left(\mathrm{YbF}^{22}\right)$ & $1 \times 10^{9}$ per sr per pulse & 290 & 1 & \\
\hline Effusive (ThO, predicted ${ }^{39}$ ) & $1 \times 10^{11}$ per sr per $\mathrm{s}$ & 540 & 2000 & 2000 \\
\hline
\end{tabular}

The minimum rotational temperatures with both helium and neon buffer gases differ by $<1 \mathrm{~K}$, despite the fact that the buffer gas cell sits at $\sim 5 \mathrm{~K}$ and $\sim 18 \mathrm{~K}$ for helium and neon, respectively. The forward velocity and divergence of the helium-based beam are slightly lower; however, both can vary as much as $30 \%$ over a single beam pulse, which is behavior not seen in our neon-based source.

In addition to time variations of beam properties within a single pulse, we find that the properties of helium-based beams also depend significantly on the location of the ablation spot, and on the pumping conditions of the buffer gas out of the beam region. Helium in the beam region can only be pumped by a large surface area adsorbent, such as activated charcoal. As the helium adsorbent fills up, the cryopumping speed begins to change, resulting in a significant change in beam properties. To keep the beam properties consistent, the adsorbent must be emptied periodically (typically every few hours for our apparatus) by heating it and then pumping the helium out of the vacuum chamber. In addition, we find that the performance of our helium-based beam is very sensitive to the amount and placement of both beam collimators and adsorbent: Incorrect placement often results in the extinction of the molecular beam, as we have observed in our apparatus and several other similar apparatus. Correct placement requires much trial and error and is not currently understood, though it has been achieved in other ThO beam test apparatus in our group. Neither the sensitivity to the ablation spot nor the charcoal placement is entirely understood.

Neon, on the other hand, is readily adsorbed by any $4 \mathrm{~K}$ surface, including neon ice, allowing for a cryopump of $>1000 \mathrm{l} \mathrm{s}^{-1}$ in the beam region of our apparatus. We have operated the neon-based molecular beam continuously, with 30 SCCM of neon flow, for over 24 hours with little increase in background pressure and no appreciable variation in beam properties. The neon-based beam is also robust to variation of the collimator geometry. We have experimented with several cell-collimator configurations, including changing the cell-collimator distance (in situ via a motion feedthrough on the apparatus), and found that for all configurations with the neon-based beam, the collimator performed as expected, and we have never had difficulty in obtaining a consistent, robust beam signal.

Another advantage of a neon-based beam is that because the cell is kept at a higher temperature, the refrigerator cooling the cell can sustain a much higher heat load. We have demonstrated operation of the neon-cooled ThO beam with nearly $10 \mathrm{~W}$ of input power from a $200 \mathrm{~Hz}$ pulsed $\mathrm{YAG}^{40}$ and achieved stable production with single-shot yields comparable to those measured with a slower repetition rate, resulting in about a factor of 10 increase in time-averaged yield compared to the data presented earlier in this paper. Further characterization of the beam with the high repetition rate is ongoing.

\subsection{Comparison to other beam sources}

Table 1 shows a comparison between the two buffer gas beam sources discussed in this paper and two common beam types, supersonic and effusive. A supersonic beam of $\mathrm{ThO}$ has been created in the past; ${ }^{41}$ however, a thorough characterization of beam properties was not performed, so we instead compare to a beam of a similar (refractory and chemically reactive) species, YbF. ${ }^{22}$ The effusive beam properties are estimated from ThO vapor pressure data. ${ }^{39}$ Which beam properties are most important depends on the application, but for a proposed ${ }^{10}$ precision spectroscopy experiment, the high count rate afforded by the increased brightness, and the long interaction time afforded by the low forward velocity, makes a buffer gas source an ideal choice.

\section{Conclusion}

We have demonstrated a general cryogenic buffer gas beam source operating in the intermediate flow regime with additional cooling from a free gas expansion, and studied its behavior in producing cold, slow, and bright molecular beams of ThO. The flux, divergence, temperature, and forward velocity of the source compare very favorably to other beam technologies, especially for chemically reactive species.

\section{Acknowledgements}

Thanks to Stan Cotreau and Dave Patterson for technical assistance; and John Barry and Edward Shuman for helpful discussions. This work was funded by a NIST Precision Measurement Grant and a grant from the National Science Foundation.

\section{References}

1 N. Ramsey, Molecular Beams, Oxford University Press, 1985.

2 G. Scoles, Atomic and Molecular Beam Methods, Oxford University Press, 1988.

3 W. D. Phillips, J. V. Prodan and H. J. Metcalf, JOSA B, 1985, 2, 1751-1767.

4 W. Ertmer, R. Blatt, J. Hall and M. Zhu, Phys. Rev. Lett., 1985, 54, 996-999. 
5 J. Prodan, A. Migdall, W. Phillips, I. So, H. Metcalf and J. Dalibard, Phys. Rev. Lett., 1985, 54, 992-995.

6 M. Zhu, C. Oates and J. Hall, Phys. Rev. Lett., 1991, 67, 46-49.

7 L. D. Carr, D. DeMille, R. V. Krems and J. Ye, New J. Phys., 2009, 11, 055049.

8 D. DeMille, Phys. Rev. Lett., 2002, 88, 067901.

9 R. V. Krems, Int. Rev. Phys. Chem., 2005, 24, 99-118.

10 A. C. Vutha, W. C. Campbell, Y. V. Gurevich, N. R. Hutzler, M. Parsons, D. Patterson, E. Petrik, B. Spaun, J. M. Doyle, G. Gabrielse and D. DeMille, J. Phys. B: At., Mol. Opt. Phys., 2010, 43, 074007.

11 J. Hudson, B. Sauer, M. Tarbutt and E. Hinds, Phys. Rev. Lett., 2002, 89, 023003.

12 V. Flambaum and M. Kozlov, Phys. Rev. Lett., 2007, 99, 150801.

13 D. DeMille, S. B. Cahn, D. Murphree, D. A. Rahmlow and M. G. Kozlov, Phys. Rev. Lett., 2008, 100, 023003.

14 A. Micheli, G. K. Brennen and P. Zoller, Nat. Phys., 2006, 2, 341-347.

15 E. S. Shuman, J. F. Barry, D. R. Glenn and D. DeMille, Phys. Rev. Lett., 2009, 103, 223001.

16 E. S. Shuman, J. F. Barry and D. DeMille, Nature, 2010, 467, $820-823$

17 H. Bethlem, G. Berden and G. Meijer, Phys. Rev. Lett., 1999, 83, $1558-1561$.

18 S. Rangwala, T. Junglen, T. Rieger, P. Pinkse and G. Rempe, Phys. Rev. A, 2003, 67, 043406.

19 R. V. Krems, W. C. Stwalley and B. Friedrich, Cold Molecules: Theory, Experiment, Applications, CRC Press, 2009, pp. 473-508.

20 S. E. Maxwell, N. Brahms, R. DeCarvalho, D. R. Glenn, J. S. Helton, S. V. Nguyen, D. Patterson, J. Petricka, D. DeMille and J. M. Doyle, Phys. Rev. Lett., 2005, 95, 173201.

21 D. Patterson and J. M. Doyle, J. Chem. Phys., 2007, 126, 154307.

22 M. R. Tarbutt, J. J. Hudson, B. E. Sauer, E. A. Hinds, V. A. Ryzhov, V. L. Ryabov and V. F. Ezhov, J. Phys. B: At., Mol. Opt. Phys., 2002, 35, 5013-5022.
23 D. Patterson, J. Rasmussen and J. M. Doyle, New J. Phys., 2009, 11, 55018.

24 J. F. Barry, E. S. Shuman and D. DeMille, arXiv:1101.4229.

25 Continuum Minilite II, $532 \mathrm{~nm}, 17 \mathrm{~mJ}$ per pulse, 6 ns pulse width.

26 We used 8-30 mesh activated coconut charcoal from Spectrum Chemical,bonded to $1 / 8^{\prime \prime}$ copper plates by Stycast 2850 FT epoxy (with catalyst $24 \mathrm{LV}$ ).

27 Cryomech PT415.

28 P. Balakrishna, B. P. Varma, T. S. Krishnan, T. R. R. Mohan and P. Ramakrishnan, J. Mater. Sci. Lett., 1988, 7, 657-660.

29 Readers new to this subject may wish to consult the review above ${ }^{19}$ for a detailed overview of buffer gas cooled beams. Additional discussion may be found in other cited works ${ }^{20,21,23,42}$.

30 Buffer gas beams can also be operated in an effusive regime, as described in previous work ${ }^{20,21,23}$.

31 Y. Sone, Molecular Gas Dynamics, Birkhäuser, Boston, 2007.

32 G. Edvinsson, L.-E. Selin and N. Aslund, Ark. Fys., 1965, 30, 283-319.

33 H. Pauly, Atom, Molecule, and Cluster Beams I, Springer-Verlag, 2000.

34 J. B. Anderson, AIChE J., 1967, 13, 1188-1192.

35 J. de la Mora and P. Riesco-Chueca, J. Fluid Mech., 1988, 195, $1-21$.

36 T. Wentink and R. Spindler, J. Quant. Spectrosc. Radiat. Transfer, 1972, 12, 1569-1590.

37 A manuscript with a detailed description of the measurement is in preparation.

38 Unpublished data with $\mathrm{Yb}$ and various alkali metals in similar apparatus.

39 R. J. Ackermann and E. G. Rauh, High Temp. Sci., 1973, 5, 463-473.

40 Litron Nano TRL $80-200,532 \mathrm{~nm}, 45 \mathrm{~mJ}$ per pulse, 8 ns pulse width.

41 V. Goncharov, J. Han, L. A. Kaledin and M. C. Heaven, J. Chem. Phys., 2005, 122, 204311.

42 D. Patterson, PhD thesis, Harvard University, 2010. 